Tohoku J. Exp. Med., 2005, 207, 243-248

\title{
Impaired Sympathetic Skin Response in Chronic Obstructive Pulmonary Disease
}

\author{
Levent Sinan Bir, Sibel ÖZkurt, ${ }^{1}$ GüNer DaloĞlu ${ }^{1}$ and Tülay Kurt ${ }^{2}$ \\ Department of Neurology, ${ }^{I}$ Department of Chest Diseases, Pamukkale University \\ Faculty of Medicine, Denizli, Turkey and \\ ${ }^{2}$ Department of Neurology, Atatürk Education and Research Hospital İzmir, \\ İzmir, Turkey
}

Bir, L.S., Özkurt, S., DaloĞLu, G. and Kurt, T. Impaired Sympathetic Skin Response in Chronic Obstructive Pulmonary Disease. Tohoku J. Exp. Med., 2005, 207 (4), 243-248 — The sympathetic skin response (SSR) is considered as one of the indexes of autonomic nervous system functions, especially related with the sudomotor function of unmyelinated sympathetic fibers. SSRs are recorded as the potentials with biphasic or multiphasic waveforms by conventional electromyography. SSRs are evaluated by measuring latency (time from the stimulus to the onset), amplitude, and area (the space under the curve of the waveform). Although dysautonomia is a feature of chronic obstructive pulmonary disease (COPD), as demonstrated by acetylcholine sweat-spot test, there are no data concerning SSR in COPD patients. In this study, we electrophysiologically investigated the sudomotor function of the sympathetic nervous system in patients with COPD. SSRs were recorded in 30 patients with COPD and 21 healthy volunteers. Normal responses were obtained from all subjects in the control group. No response was observed in three patients with COPD. The mean latency, amplitude and area values of the potentials recorded of the remaining 27 patients were compared to the control. The mean latency was longer $(p<0.01)$ and the mean amplitude and area values were lower $(p=$ $0.012, p=0.021$, respectively) in the patients compared to the control. We also demonstrated significant correlations between the latency, amplitude, or area values of the SSR and two parameters of pulmonary function tests forced expiratory volume one second/ forced vital capacity (FEV1/FVC) and FEV1/FVC \%. In conclusion, SSR is impaired in patients with COPD, which indicates the dysfunction of the sympathetic nervous system. Furthermore, the degree of impairment in SSR may reflect the severity of airway obstruction in patients with COPD. - chronic obstructive pulmonary disease; sympathetic skin response; autonomic nervous system; dysautonomia; electromyography

(C) 2005 Tohoku University Medical Press

In chronic obstructive pulmonary disease (COPD) and asthma, airway obstruction is mainly determined by the contractile state of smooth muscle cells in the airway, the thickness of the airway walls, and elastic recoil of the lungs and hypersecretion of mucus. Autonomic nerves can influence the airway caliber via their effects on airway smooth muscles, bronchial vessels and

Received July 13, 2005; revision accepted for publication September 7, 2005.

Correspondence: Dr. Levent Sinan Bir, Pamukkale Üniversitesi Tıp Fakültesi Hastanesi, Kınıklı Kampüsü, Nöroloji Anabilim Dalı B105, Kınıkl1- 20075 Denizli Turkey.

e-mail: sbir@pamukkale.edu.tr 
mucous glands, and may therefore contribute to the narrowing of the airway in COPD and asthma; but there is no direct evidence supporting the presence of abnormal autonomic control in COPD (de Jongste et al. 1991). Although the airway smooth muscle cell possesses many relaxing beta- 2 receptors, it receives only a few sympathetic nerves (Davis and Kannan 1987). It was also shown that pulmonary sympathetic denervation by high thoracic epidural anesthesia did not increase airway resistance in patients with COPD (Groeben et al. 1995). Inflammation and oxidative stress seem to be more important mechanisms in the pathogenesis of COPD (Ichinose 2003).

On the other hand, many studies investigating the autonomic nervous system (ANS) functions by different methodologies indicated the dysautonomia in COPD (Stewart et al. 1991; Volterranni et al. 1994; Pagani et al. 1996). Stewart et al. (1994) demonstrated the presence of sympathetic autonomic neuropathy in patients with COPD by acetylcholine sweat-spot test and emphasized that subclinical autonomic neuropathy is a feature of hypoxaemic COPD.

In this study we aimed to investigate the sudomotor function of the sympathetic nervous system in patients with COPD electrophysiologically. To our knowledge there are no data concerning sympathetic skin response (SSR) in COPD patients. This test especially evaluates the sudomotor function of unmyelinated sympathetic fibers (Fagius and Wallin 1980). SSR is a simple, reproducible test evaluating the polysynaptic reflex having diverse afferents, a common efferent pathway through the spinal cord, pre- and postganglionic sympathetic fibers and sweat glands as effectors. The reflex is coordinated in the posterior hypothalamus or upper brainstem reticular formation (Arunodaya and Taly 1995). The electrophysiologic technique for obtaining SSR has been defined to quantify the changes in voltage which have been attributed to sudomotor activity. It is measured from skin after electrical stimulation (Shahani et al. 1984). The transient changes in the skin electrical resistance have been attributed to the movement of ions across the membrane of skin sweat gland cells (Lader and Montagu 1962).

\section{Materials And Methods}

\section{Patients}

Thirty patients with COPD (21 men, 9 women), and 21 healthy volunteers as the control group (15 men, 6 women) were enrolled in this study. Age, sex and disease duration of each subject were recorded. As it was reported that the SSR latency was correlated well with height (Oh 1993), the heights of the subjects were also recorded. None of the subjects were taking any systemic medication and had any other disease that could affect the SSR recordings. None of the subjects were using inhaler corticosteroid. Inhaler anticholinergics and/or sympathomimetics that were used by some patients were terminated one day before the tests. All of the COPD patients were ex-smoker, and all of the subjects were non-smoker in the control group. According to clinical and pulmonary function test (PFT) values, 6 patients had mild, 11 had moderate, 13 had severe COPD.

Peripheral neuropathy and other neurological disorders were excluded by neurological examination, and normal motor and sensory functions of nervi medianus were revealed by electroneurographic examination in all of the subjects. Informed consent was taken from all of the subjects. All experiments were performed in compliance with the relevant laws.

\section{Methods}

This study was performed in a semi-darkened room at an ambient temperature of $23^{\circ} \mathrm{C}$ while the patients were in relaxed and supine position at the same hours of the day (12:00-1:00 p.m.). SSR recordings were performed by a Medelec Premiere Plus electromyography (EMG) machine. Stainless steel disk electrodes in $6 \mathrm{~mm}$ diameter were used for the recordings. The active electrode was attached to the palm, and the reference electrode was attached to the dorsum of the hand, bilaterally. Band pass filters were set between 1 and 1,000 Hz and the sensitivity was 0.5 and $1 \mathrm{mV}$. SSR recordings were obtained from both hands using two channels after one pulse. Pulses in 0.1 milliseconds duration and $30 \mathrm{~mA}$ intensity were applied to the skin of both wrists exciting nervi medianus and totally four SSR recordings were obtained from each subject. The latency and amplitude measurements in SSR were performed manually by the cursors of the EMG machine. Latency was measured with the sensitivity of milliseconds from the onset of stimulus artifact to the onset of skin response and recorded as seconds. The amplitude was measured in millivolts 
from peak to peak. The area under the negative component of SSR was recorded automatically in millivolts second.

After SSR study, PFT and arterial blood gas analysis were performed in each patient with COPD on the same day. PFT was done with a spirometry $\left(\mathrm{MIR}^{\circledR}\right)$ that used dry system infrared interruption method. Forced vital capacity (FVC), FVC\%, forced expiratory volume one second (FEV1), FEV1\%, FEV1/FVC, FEV1/FVC\% and peak expiratory flow (PEF) and $\mathrm{PEF} \%$ values of the patients were recorded. $\mathrm{pH}, \mathrm{pCO}_{2}, \mathrm{pO}_{2}$ and saturation of $\mathrm{O}_{2}$ values were obtained by blood gas analysis system in each patient.

\section{Statistics}

Statistical analysis was done using Statistical Package for Social Sciences (SPSS) for Microsoft Windows. For each subject, the mean values of the parameters obtained from the best four responses were calculated. The groups were compared for the mean age, the mean latency; the mean amplitude and the mean area under the negative component of SSR values by student $t$-test. The male/female distribution of the groups was compared by chi-square test. The correlation analyses between the SSR parameters (latency, amplitude and area under the negative component) and PFT parameters or the values of arterial blood gases were performed by Pearson test.

\section{RESUlts}

The mean ages of COPD and control group were $60.9 \pm 9.54$ years and $57.28 \pm 7.85$ years, respectively $(p=0.160)$. The mean height of the subjects were $163.6 \pm 6.7 \mathrm{~cm}$ and $161.61 \pm 5.56$ $\mathrm{cm}$, respectively $(p=0.275)$. The gender distributions of the two groups were also found to be comparable $(p>0.05)$. The mean duration of disease was $69.88 \pm 0.52$ months in the patient group. Normal responses could be obtained from all of the subjects in control group. The recorded potentials were biphasic or triphasic (Fig. 1A). In three patients with COPD no response could be observed (Fig. 1C). As shown in Fig. 1B, the responses obtained from the patients with COPD were generally in small amplitude and had prolonged latency. The calculated latency, amplitude and area values of the SSR potentials of the rest 27 patients were compared to the control group.

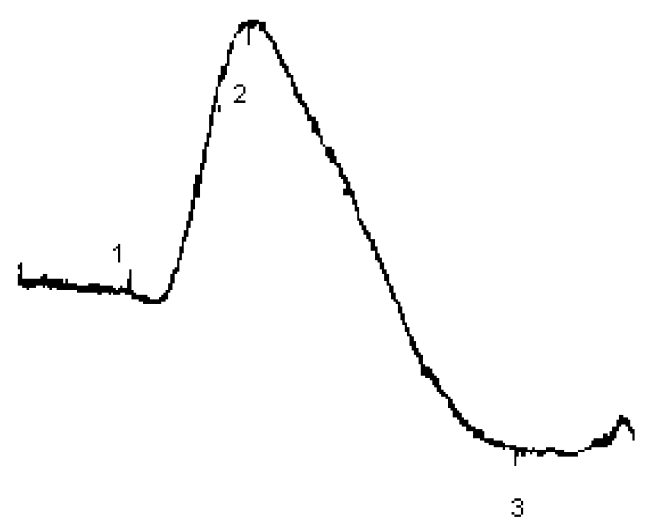

A

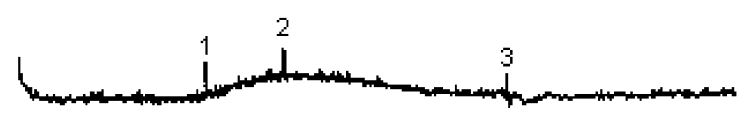

B

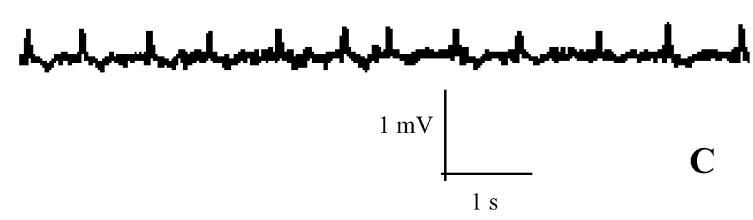

Fig. 1. Examples of SSR from control and patient groups. A: A typical SSR obtained from control group. (1) represents the starting point of the potential, (2) is the peak point and (3) is the end point. B: SSR in small amplitude and prolonged latency obtained from a patient with COPD. C: Absent SSR in another patient with COPD. Only ECG artefact is seen.

The mean latency, amplitude and the area under the negative component values of SSRs were summarized in Table 1. The mean latency of the potentials was longer $(p<0.01)$, and the mean amplitude and area values were lower $(p=0.012$, $p=0.021$, respectively) in patient group.

Results of PFT and blood gas analysis of the patients were summarized in Table 2. A meaningful correlation at $0.05 p$ level among FEV1/FVC and latency $(r=-0.468, p=0.016)$, FEV1/FVC $\%$ and latency $(r=-0.640, p<0.01)$, the amplitude and FEV1/FVC ( $r=0.408, p=0.039)$, the area and FEV1/FVC $(r=0.401, p=0.043)$, the 
TABLE 1. Mean latency, amplitude and area under the negative component values of SSR in COPD and control groups (mean \pm S.D.)

\begin{tabular}{cccc}
\hline SSR parameters & Latency $(\mathrm{s})^{*}$ & Amplitude $(\mathrm{mV})^{*}$ & $\begin{array}{c}\text { Area under the negative } \\
\text { component of SSR (mVs) }\end{array}$ \\
\hline COPD $(n=27)$ & $1.66 \pm 0.21$ & $2.89 \pm 3.42$ & $7.44 \pm 8.05$ \\
Control $(n=21)$ & $1.50 \pm 0.19$ & $6.06 \pm 4.63$ & $13.55 \pm 10.16$ \\
\hline${ }^{*} p<0.05$. & & &
\end{tabular}

TABLE 2. Mean and standard deviation and correlation values of pulmonary function tests and blood gas analysis (with SSR parameters) of the patient group

\begin{tabular}{|c|c|c|c|c|c|c|c|}
\hline \multirow{3}{*}{ Parameter } & \multirow{3}{*}{ Mean \pm S.D. } & \multicolumn{6}{|c|}{ Pearson correlation } \\
\hline & & \multicolumn{2}{|c|}{ SSR latency } & \multicolumn{2}{|c|}{ SSR amplitude } & \multicolumn{2}{|c|}{ SSR area } \\
\hline & & $r$ & $p$ & $r$ & $p$ & $r$ & $p$ \\
\hline FVC (lt) & $1.65 \pm 0.67$ & 0.306 & 0.129 & 0.073 & 0.722 & 0.117 & 0.568 \\
\hline $\mathrm{FVC} \%$ & $49.44 \pm 18.04$ & 0.013 & 0.957 & 0.226 & 0.352 & 0.258 & 0.287 \\
\hline FEV1 (liter/min) & $1.27 \pm 0.56$ & 0.105 & 0.610 & 0.249 & 0.219 & 0.285 & 0.159 \\
\hline FEV1\% & $46.50 \pm 22.38$ & -0.184 & 0.451 & 0.418 & 0.750 & 0.433 & 0.064 \\
\hline FEV1/FVC & $77.88 \pm 14.12$ & -0.468 & $0.016^{*}$ & 0.408 & $0.039^{*}$ & 0.401 & $0.043^{*}$ \\
\hline FEV1/FVC\% & $97.65 \pm 30.02$ & -0.640 & $0.003^{*}$ & 0.523 & $0.022^{*}$ & 0.519 & $0.023^{*}$ \\
\hline PEF (liter/min) & $3.25 \pm 1.45$ & 0.147 & 0.475 & 0.196 & 0.338 & 0.208 & 0.308 \\
\hline PEF\% & $39.42 \pm 16.30$ & -0.065 & 0.791 & 0.343 & 0.150 & 0.336 & 0.160 \\
\hline $\mathrm{pH}$ & $7.38 \pm 0.05$ & 0.144 & 0.493 & 0.110 & 0.593 & 0.105 & 0.609 \\
\hline $\mathrm{pO}_{2}(\mathrm{mmHg})$ & $69.22 \pm 14.76$ & 0.101 & 0.632 & -0.127 & 0.536 & -0.138 & 0.500 \\
\hline $\mathrm{pCO}_{2}(\mathrm{mmHg})$ & $43.51 \pm 9.85$ & -0.292 & 0.167 & 0.168 & 0.320 & 0.081 & 0.167 \\
\hline $\mathrm{O}_{2}$ sat. $(\%)$ & $90.11 \pm 9.05$ & 0.222 & 0.298 & -0.121 & 0.574 & -0.129 & 0.546 \\
\hline
\end{tabular}

${ }^{*} p<0.05$.

amplitude and FEV1/FVC $\%(r=0.523, p=$ $0.022)$, the area and FEV1/FVC \% $(r=0.519, p=$ $0.023)$ were detected. There were no significant correlations among the parameters of SSR and the duration of the disease. Also no meaningful correlations were detected among the arterial blood gas results and SSR parameters.

\section{Discussion}

In the current study we detected impairment in sympathetic skin responses in COPD patients. While SSR could be obtained from all of the control subjects, it could not be obtained from three patients with COPD. In the rest 27 patients, the mean latency of the potentials was longer, and the average amplitude and the area were lower com- pared to the control group. We propose that the sympathetic nervous system is affected in COPD. We also showed that some parameters (FEV1/FVC and FEV1/FVC\%) of PFT reflecting the degree of airway obstruction were correlated with sympathetic nerve function on sweat glands. It is impossible to detect the source of sympathetic dysfunction in CODP by SSR. Probably hypoxia causes sympathetic autonomic neuropathy.

Gross and Skorodin (1984) studied the relative contribution of sympathetic and parasympathetic mechanisms in airway obstruction in patients with emphysema, by applying supramaximal doses of anticholinergic and adrenergic agents. They concluded that parasympathetic activity is a dominant reversible component of 
airway obstruction. Groeben et al. (1995) detected that the sympathetic blockage induced by high thoracic epidural anesthesia did not cause any evident increase in airway resistance in COPD patients. Primary neural control of airway function is via parasympathetic pathways (Bleecker 1986) and there is no convincing data for the presence of abnormal adrenergic control in the airways of patients with COPD (de Jongste et al. 1991). According to the authors, postjunctional factors such as airway thickening, decreased elastic recoil of the lungs, mucus hypersecretion and increased reflex vagal activation may well suffice to explain airway obstruction. They also indicate that many aspects of airway caliber regulation by the ANS are still unclear and require further study. Based on these findings summarized above, sympathetic dysfunction has no major role in COPD pathogenesis, but COPD might cause sympathetic dysfunction.

Abnormalities in both parasympathetic and sympathetic nervous system in COPD patients were demonstrated in many studies. Depressed heart rate variability (HRV) responses to sympathetic and vagal stimuli were detected in a study (Volterrani et al. 1994). Stewart et al. (1991) defined definite autonomic dysfunction (35\%) and early autonomic neuropathy (47\%) in COPD patients, based on the findings in heart rate responses to valsalva maneuver, deep breathing and postural change (mainly of parasympathetic function), and blood pressure responses to postural change and sustained handgrip (mainly of sympathetic origin). The authors emphasized that intraneural hypoxemia was recognized as a pathogenic mechanism in diabetic neuropathy and a similar pathophysiological process might occur in COPD. In another study, they also detected impaired sweating in patients with COPD by acetylcholine sweat-spot test (Stewart et al. 1994). It was also stated that sympathetic excitatory modulation of the sinoatrial node was depressed in COPD patients (Pagani et al. 1996). Increased vagal activity that was evident at nights, and decreased urinary noradrenaline and adrenaline levels were detected in COPD patients, and it was hypothesized that this ANS imbalance might underlie the nocturnal dyspnea in patients with chronic airflow obstruction (Postma et al. 1985).

All these studies summarized above indicate ANS dysfunction. Detection of ANS dysfunction in COPD is important. It may develop as secondary to the disease process, but in time, it may cause some negative effects on the progression of the disease and may create a vicious circle. It was indicated that patients with ANS dysfunction were more hypoxemic and had lower FEV1 and prolonged QT. It was also demonstrated that ANS dysfunction in patients with COPD was related with ventricular arrhythmias (Sharp et al. 1998) and patients with COPD who had ANS dysfunction were more prone to edema because of reduction in urine outflow compared to the patients with intact ANS functions (Stewart et al. 1995).

In conclusion, impairment of SSR was demonstrated electrophysiologically in patients with COPD. This finding indicates the sudomotor dysfunction of ANS in COPD. We also demonstrated that severity of the airway obstruction was correlated with the sudomotor dysfunction of sympathetic nervous system in patients with COPD. We recommend this cheap and easy method in detection and evaluation of the sympathetic nervous system dysfunction in patients with COPD.

\section{References}

Arunodaya, G.R. \& Taly, A.B. (1995) Sympathetic skin response: a decade later. J. Neurol. Sci., 129, 81-89.

Bleecker, E.R. (1986) Cholinergic and neurogenic mechanism in obstructive airways disease. Am. J. Med., 81, 93-102.

Davis, C. \& Kannan, M.S. (1987) Sympathetic innervation of human tracheal and bronchial smooth muscle. Respir. Physiol., 68, 53-61.

de Jongste, J.C., Jongejan, R.C. \& Kerrebijn, K.F. (1991) Control of airway caliber by autonomic nerves. Am. Rev. Respir. Dis., 143, 1421-1426.

Fagius, J. \& Wallin, B.G. (1980) Sympathetic reflex latencies and conduction velocities in normal men. J. Neurol. Sci., 47, 433-448.

Groeben, H., Schwalen, A., Irsfeld, S., Lipfert, P. \& Hopf, H.B. (1995) Pulmonary sympathetic denervation does not increase airway resistance in patients with chronic obstructive pulmonary disease. Acta Anaesthesiol. Scand., 39, 523-526.

Gross, N.J. \& Skorodin, M.S. (1984) Role of the parasympathetic system in airway obstruction due to emphysema. $N$. Engl. J. Med., 317, 421-425.

Ichinose, M. (2003) Inflammatory mechanisms in bronchial astma and COPD. Tohoku J. Exp. Med., 200, 1-6.

Lader, M.H. \& Montagu, J.D. (1962) The pseudogalvanic re- 
flex: a pharmacological study of the peripheral mechanism. J. Neurol. Neurosurg. Psych., 25, 126-133.

Oh, S.J. (1993) Clinical Electromyography: Nerve Conduction Studies, 2nd ed., Williams and Wilkins, Baltimore, 270.

Pagani, M., Lucini, D., Pizzinelli, P., Sergi, M., Bosisio, E., Mela, G.S. \& Malliani, A. (1996) Effects of aging and of chronic obstructive pulmonary disease on RR interval variability. J. Auton. Nerv. Syst., 59, 125-132.

Postma, D.J., Keyzer, J.J., Koeter, G.H., Sluiter, H.J. \& De Vries, K. (1985) Influence of the parasympathetic and sympathetic nervous system on nocturnal bronchial obstruction. Clin. Sci., 69, 251-258.

Shahani, B.T., Halperin, J.J., Boulu, P. \& Cohen, J. (1984) Sympathetic skin response - a method of assessing unmyelinated axon dysfunction in peripheral neuropathies. $J$. Neurol. Neurosurg. Psych., 47, 536-542.

Sharp, D.S., Masaki, K., Burchfiel, C.M., Yano, K. \& Schatz, I.J. (1998) Prolonged QTc interval, impaired pulmonary func- tion, and a very body mass jointly predict all cause mortality in elderly men. Ann. Epidemiol., 8, 99-106.

Stewart, A.G., Waterhouse, J.C. \& Howard, P. (1991) Cardiovascular autonomic nerve function in patients with hypoxemic chronic obstructive pulmonary disease. Eur. Respir. J., 4, 1207-1214.

Stewart, A.G., Marsh, F., Waterhouse, J.C. \& Howard, P. (1994) Autonomic nerve dysfunction in COPD as assessed by the acetylcholine sweat-spot test. Eur. Respir. J., 7, 1090-1095.

Stewart, A.G., Waterhouse, J.C., Billings, C.G., Baylis, P.H. \& Howard, P. (1995) Hormonal, renal, and autonomic nerve factors involved in the excretion of sodium and water during dynamic salt and water loading in hypoxaemic chronic obstructive pulmonary disease. Thorax, 50, 838-845.

Volterrani, M., Scalvini, S., Mazzuero, G., Lanfranchi, P., Colombo R., Clark, A.L. \& Levi, G. (1994) Decreased heart rate variability in patients with chronic obstructive pulmonary disease. Chest, 106, 1432-1437. 